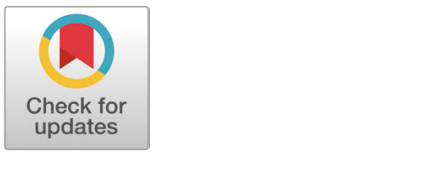

Received: Nov 19, 2019

Revised: Jan 14, 2020

Accepted: Mar 15, 2020

*Corresponding author

Hyun-Jeong Lee

Division of Dairy Science,

National Institute of Animal Science,

Cheonan 31000, Korea.

Tel: +82-41-580-3396

E-mail: Jeong73@korea.kr

Copyright $(02020$ Korean Society of Animal Sciences and Technology.

This is an Open Access article distributed under the terms of the Creative Commons Attribution Non-Commercial License (http:// creativecommons.org/licenses/by$\mathrm{nc} / 4.0 /$ ) which permits unrestricted non-commercial use, distribution, and reproduction in any medium, provided the original work is properly cited.

ORCID

Jin Young Jeong

https://orcid.org/0000-0002-8670-7036

Youl-Chang Baek

https://orcid.org/0000-0003-4454-5339

Sang Yun Ji

https://orcid.org/0000-0001-7235-3655

Young Kyun Oh

https://orcid.org/0000-0002-5817-1748

Soohyun Cho

https://orcid.org/0000-0002-8073-8771

Hyun-Woo Seo

https://orcid.org/0000-0002-7587-0612

Minseok Kim

https://orcid.org/0000-0002-8802-5661

\title{
Nuclear magnetic resonance-based metabolomics analysis and characteristics of beef in different fattening periods
}

\author{
Jin Young Jeong ${ }^{1}$, Youl-Chang Baek ${ }^{1}$, Sang Yun Ji', Young Kyun Oh', \\ Soohyun $\mathrm{Cho}^{2}$, Hyun-Woo Seo ${ }^{2}$, Minseok Kim ${ }^{3}$ and Hyun-Jeong Lee ${ }^{1,4 *}$ \\ ${ }^{1}$ Animal Nutrition \& Physiology Team, National Institute of Animal Science, Wanju 55365, Korea \\ ${ }^{2}$ Animal Production Utilization Division, National Institute of Animal Science, Wanju 55365, Korea \\ ${ }^{3}$ Department of Animal Science, College of Agriculture and Life Science, Chonnam National University, \\ Gwangju 61186, Korea \\ ${ }^{4}$ Division of Dairy Science, National Institute of Animal Science, Cheonan 31000, Korea
}

\section{Abstract}

Beef quality is influenced by the fattening period. Therefore, meat metabolomics profiles from the different fattening periods (e.g., short-term vs. long-term) were analyzed for identify potential indicators using nuclear magnetic resonance. Additionally, blood, free fatty acid, sensory, and mineral compositions in Korean steers were determined. Blood, free fatty acid, and mineral concentrations showed significant differences between short-term and long-term groups that were fed different diets. However, there were no sensory differences in the two fattening groups. Additionally, the metabolic profiles of meats were clearly separated based on multivariate orthogonal partial least square discriminant analysis. Six metabolites of variable importance in the projection plot were identified and showed high sensitivity as candidate markers for meat characteristics. In particular, lactate, carnosine, and creatine could be directly linked to scientific indicators of the fattening stage (31 vs. $28 \mathrm{mo}$ ) of meat. Our findings suggest that the metabolomics approach could be a powerful method for the detection of novel signatures underlying the managing period of beef.

Keywords: Beef, Metabolomics, Short-term, Long-term, Fattening period

\section{INTRODUCTION}

Meat is an essential food for growth and health promotion in humans, and the consumption of beef has consistently increased across the world [1]. Improving beef quality and reducing management costs will bring about economic benefits and benefit the meat production industry. Recently, meat characterization has been widely carried out based on the levels of metabolites [2,3]. In particular, factors such as differences in fattening periods may provide a clue for refining beef quality through metabolomics profiling.

Metabolomics profiles have been used to identify potential indicators in various biological systems. 
Hyun-Jeong Lee

https://orcid.org/0000-0002-2312-9048

Competing interests

No potential conflict of interest relevant to

this article was reported.

Funding sources

This research was supported by a grant the Research Program for Agriculture Science \& Technology Development (Project No.

PJ012031022018) of the National Livestock Research Institute, Rural Development Administration, Korea.

Acknowledgements

Not applicable.

Availability of data and material Upon reasonable request, the datasets of this study can be available from the corresponding author.

Authors' contributions

Conceptualization: Jeong JY, Lee HJ.

Formal analysis: Baek YC.

Methodology and Software: Seo HW.

Validation: Ji SY.

Investigation: Cho S

Writing - original draft: Jeong JY.

Writing - review \& editing: Oh YK, Kim M, Lee HJ.

Ethics approval and consent to participate All animal experiments were approved by the Institutional Animal Care and Use Committee of the National Institute of Animal Science, Korea (Approval number : NIAS20191665). We have read and understood your journal's policies, and we believe that neither the manuscript nor the study violates any of these. This manuscript has not been published and is not under consideration by another journal.
Nuclear magnetic resonance (NMR), spectroscopy, and mass spectrometry (MS) are powerful tools used for biomarker screening [4,5]. NMR is a commonly used technique to identify and quantify molecules without the need for reference standards. Moreover, metabolic profiling provides a new opportunity to study the influence of different conditions.

In the last few decades, there has been a renewed interest in improving meat production and manipulating several factors to improve meat quality, nutrition, and production costs. Intrinsic characteristics of taste, fatty acid, and mineral compositions may differ according to the steer diet and fattening period [6,7]. Minerals have a positive effect on the steers, such as high digestibility, mineralization of bones and teeth, development of nervous system and muscle, and activation of enzymes in vivo [8]. Free fatty acid compositions are affected by breed type, feeding system, and intramuscular fat, resulting in differences in sensory characteristics [9]. Thus, the taste of beef meat is affected by meat quality including the fatty acid compositions [10]. Good beef taste is the primary consumer demand and beef taste may be enhanced using varied combinations of fatty acids, minerals, and sensory-related metabolites. A better understanding of the meat quality from traditional analysis and metabolomics profiling depending on managing systems is required to reduce the production costs and enhance the meat quality. Thus, the objective of this study was to evaluate and compare the characteristics of beef meat between short-term and long-term fattening periods using metabolomics analysis, and through assessment of free fatty acid content, mineral composition, and taste score.

\section{MATERIALS AND METHODS}

\section{Animals and sample preparation}

All experimental studies were reviewed by the National Institute of Animal Science Institutional Animal Care and Use Committee (NIASIACUC), Korea.

Twenty-two samples of beef meat were collected from the NIAS at post-mortem. Meat samples were obtained from short-term $(n=12)$ and long-term $(n=10)$ fattened Korean steers (i.e., Hanwoo) at approximately 28 and 31 months, respectively. Calves were weaned at 3 months, and fed with concentrates (30\%) and roughage (70\%) until they reached 6 months of age. Bulls were castrated between 6 and 7 months of age. For the long-term (LT) fattening period, beef steers were fed with concentrates that consisted of $15 \%$ crude protein (CP)/70\% total digestible nutrient (TDN) until 13 months of age, 14\% CP/73\% TDN until 21 months of age, and 13\% CP/76\% TDN until the final fattening stage at 31 months. For the short-term (ST) fattening period, beef was fed with 18\% CP/74\% TDN until 13 months, and 16\% CP/76\% TDN until 19 months, and 14\% CP/80\% TDN until the final fattening stage at 28 months. Roughage was offered ad libitum and free access to water was arranged during the entire period. After slaughter and a $24-\mathrm{h}$ chill period at $2{ }^{\circ} \mathrm{C}$, loin (longissimus dorsi) samples were taken from the dorsal area of the 13th rib and the cold carcass weight was measured. The marbling score (MS) was estimated on the left side between the last rib and first lumbar vertebra in accordance with the beef marbling standard (BMS). Meat samples were graded $1+$ as marbling scores (MS; 6-7 on the scale of 1-9). Meat in the two meat groups (ST and LT) was frozen using liquid nitrogen and stored at $-80^{\circ} \mathrm{C}$. All samples for metabolomics profiling, mineral analysis, taste evaluation, and free fatty acid concentrations were pulverized in a mortar containing liquid nitrogen.

\section{NMR analysis}

Beef meat samples $(25 \mathrm{mg})$ were used for metabolomics profiling by NMR. Briefly, samples were transferred to 4-mm NMR nanotubes with $25 \mu \mathrm{L}$ deuterium oxide containing $2 \mathrm{mM}$ 3-(trimeth- 
ylsilyl) propionic-2,2,3,3- $\mathrm{d}_{4}$ acid sodium salt (TSP- $\mathrm{d}_{4}$; Sigma Aldrich, St. Louis, MO, USA) as an internal standard. The NMR spectra for meat samples were acquired by a $600 \mathrm{MHz}$ Agilent NMR spectrometer (Agilent Technologies, Palo Alto, CA, USA) with a 4-mm gHX NanoProbe for high-resolution magic angle spinning at Pusan National University in Korea. Data were collected at a spinning rate of 2,000 Hz. A Carr-Purcell-Meiboom-Gill pulse sequence was used to reduce the background signals of water and macromolecules in the tissues. The ${ }^{1} \mathrm{H}-\mathrm{NMR}$ spectra were measured using $13 \mu \mathrm{s}$ of a $90^{\circ}$ pulse, $0.065 \mathrm{~s}$ of bigtau, $2 \mathrm{~s}$ of relaxation delay, $1.704 \mathrm{~s}$ of acquisition time, and $10 \mathrm{~min} 20 \mathrm{~s}$ of total acquisition time. The TSP- $\mathrm{d}_{4}$ peak at $0.0 \mathrm{ppm}$ was used for reference to calibrate the chemical shifts. Assignment of spectra and quantification of metabolites were accomplished by Chenomx NMR suite 7.1 software (Chenomx, Edmonton, AB, Canada).

\section{Sensory evaluation by electronic tongue analysis}

The sensory evaluations were assessed using an electronic tongue (Astree, Alpha MOS, Toulouse, France) coupled with an automatic sample analyzer. The specific taste sensor module was composed of seven sensors (Sensor array \#5) and a reference electrode $(\mathrm{Ag} / \mathrm{AgCl})$. The seven sensors comprised SRS (sourness), GPS (metallic), STS (saltiness), SPS (spiciness), UMS (umami), SWS (sweetness), and BRS (bitterness). SPS and GPS sensors are used as a standard for calibration. Equivalent amounts of $40 \mathrm{~g}$ meat were dispersed into $120 \mathrm{~mL}$ of distilled water, homogenized for $60 \mathrm{~s}$, and then filtered using PDVF syringe filter $(4.5 \mu \mathrm{m})$. Acquisition time was set at $120 \mathrm{~s}$ at $25^{\circ} \mathrm{C}$ and analysis time at $180 \mathrm{~s}$. Five replicates were analyzed. The data were expressed as means \pm sem.

\section{Mineral analysis}

The mineral content in the meat was processed using a microwave oven (Milestone SRL, Sorisole, Italy) with a heating program with a $15 \mathrm{~min}$ ramp time and $10 \mathrm{~min}$ hold time at $1,500 \mathrm{~W}$. The temperature and maximum pressure were set at $220^{\circ} \mathrm{C}$ and 110 bar, respectively. The mineral elements such as $\mathrm{Ca}, \mathrm{Cu}, \mathrm{Fe}, \mathrm{K}, \mathrm{Na}, \mathrm{Mg}, \mathrm{Mn}, \mathrm{P}$, and $\mathrm{Zn}$ were determined using inductively coupled plasma-optical emission spectrometer (ICP-OES, PerkinElmer optima 4300 DV, Shelton, USA) as previously described. Briefly, meat samples (approximately $0.3 \mathrm{~g}$ ) were weighed and diluted with $\mathrm{NO}_{3}(2 \%-3 \%)$. Argon gas (99.9\%) was used for plasma generation (15 L/min) and nebulization $(0.55 \mathrm{~L} / \mathrm{min})$, as an auxiliary gas $(1.5 \mathrm{~L} / \mathrm{min})$ as well as to pressurize the chamber. The sample uptake rate, rinse time, pump rate, integration time, and replicate number were $1 \mathrm{~mL} / \mathrm{min}, 35 \mathrm{~s}, 15$ $\mathrm{rpm}, 30 \mathrm{~s}$, and 3, respectively. The data were analyzed using GraphPad Prism (GraphPad Software [Ver. 5.03, 2009], San Diego, CA, USA). All the analyses were done in three replicates. Mineral concentrations were expressed as ppm $(\mathrm{mg} / \mathrm{L})$ and standard error of the mean.

\section{Blood analyses}

Blood samples $(20 \mathrm{~mL})$ were collected from the jugular vein into a Vacutainer before slaughter. The plasma was collected after centrifugation at $3,000 \times \mathrm{g}$ at $4^{\circ} \mathrm{C}$ for $15 \mathrm{~min}$. The serum samples were divided into portions $(1 \mathrm{~mL})$ and stored at $-20^{\circ} \mathrm{C}$. Total lipid was analyzed using the colorimetric method (spectrophotometer, Agilent 8453, Germany) with total lipid reagents (Medicos, USA). Insulin was measured by the ELISA method (microplate reader, VERSA Max, USA) with the bovine insulin ELISA kit (Mercodia, Sweden). Total cholesterol, glucose, high-density lipoprotein (HDL) cholesterol, low-density lipoprotein (LDL) cholesterol, and triglyceride were determined using the enzymatic colorimetric CHOL2 assay, enzymatic reference method with glucose HK Gen.3, homogeneous enzymatic colorimetric assay using HDL-C plus $3^{\text {rd }}$ generation, homogeneous enzymatic LDL-cholesterol assay, and triglyceride colorimetric assay, respectively, by Cobas 8000 c702 analyzer (Roche Diagnostics, Mannheim, Germany). 


\section{Fatty acid analysis}

Fatty acids in the beef samples were measured using chloroform:methanol (2:1, v/v), according to the procedure [11]. The total lipid extract was methylated as previously described [12]. The fatty acid methyl esters were analyzed using a gas chromatography (Varian 3600, USA) coupled with a fused silica capillary column, omegawax $320(30 \mathrm{~m} \times 0.32 \mathrm{~mm}$ ID, $0.25 \mu \mathrm{M}$ film thickness). Briefly, the oven temperature was set at $200^{\circ} \mathrm{C}$ and the carrier gas velocity was $25 \mathrm{~cm} / \mathrm{s}$. The injection port was set at $250^{\circ} \mathrm{C}$ and the detector was maintained at $300^{\circ} \mathrm{C}$. Results of total fatty acid compositions were expressed as percentages based on detected total peak area. The measured fatty acids such as myristic acid (C14:0), palmitic acid (C16:0), palmitoleic acid (C16:1n7), stearic acid (C18:0), oleic acid (C18:1n9), vaccenic acid (C18:1n7), linoleic acid (C18:2n6), $\gamma$-linolenic acid (C18:3n6), $\alpha$-linolenic acid (C18:3n3), eicosenoic acid (C20:1n9), arachidonic acid (C20:4n6), adrenic acid (C22:4n6), total saturated fatty acids (SFA), unsaturated fatty acids (USFA), monounsaturated fatty acids (MUFA), polyunsaturated fatty acids (PUFA), omega-3 ( $\omega 3)$, omega-6 ( $\omega 6)$, ratio between omega -6 and omega-3 ( $\omega 6 / \omega 3)$, rate of MUFA and SFA, and proportion between PUFA and SFA were calculated using appropriate components.

\section{Statistical analyses}

Metabolic profiles from multivariate methods were analyzed. To obtain meat metabolic profiles, NMR spectra were binned with a 0.001-ppm binning size. The binned spectra were normalized to the total area and aligned using the icoshift algorithm of MATLAB R2013b (Mathworks, Natick, MA, USA). The binning results were imported into the SIMCA software. The peak areas of metabolites were subjected to partial least squares-discriminant analysis (PLS-DA; SIMCA version 14, Umetrics, Umea, Sweden) to visualize cluster separation between short-term and long-term fattening periods. The statistical significance $(p<0.05)$ of metabolite concentrations, and mineral, sensory, and fatty acid compositions were evaluated by unpaired $t$-tests. PLS-DA was performed on Pareto-scaled data to visualize general clustering of all samples on the scores plot, which was defined with $95 \%$ confidence intervals. Variable importance in projection (VIP) plots were also utilized as potential indicators. VIP values greater than 1.0 were considered important in discriminating between groups. Analyses of the metabolites and characterization data were performed using Excel 2016 (Microsoft) and GraphPad Prism ver. 5.03 (GraphPad Software).

\section{RESULTS AND DISCUSSION}

\section{Carcass traits, blood analysis, and free fatty acid compositions}

Carcass characteristics, blood, and free fatty acid compositions were compared between ST and LT samples, as shown in Table 1. Backfat thickness was higher $(p<0.001)$ in ST than in LT samples, while the meat yield grade was significantly decreased $(p<0.001)$ in ST compared with LT. Cold carcass weight and marbling score were not statistically different between ST and LT samples. Glucose, insulin, HDL and LDL-cholesterol, total cholesterol, and total lipid showed significant differences between the two different fattening groups. Glucose $(p=0.0019)$, HDL- $(p=0.0093)$ and LDL-cholesterol $(p<0.001)$, total cholesterol $(p=0.0009)$, and total lipid $(p=0.0058)$ were significantly increased in ST. However, insulin $(p=0.0015)$ had higher concentrations in LT than in ST samples. There was no significant difference in triglyceride concentrations between the two groups. The concentrations of $\gamma$-linolenic acid (GLA, $p=0.0367$ ), arachidonic acid (ARA, $p=0.0053$ ), and adrenic acid (AdA, $p=0.0146$ ) showed higher fatty acid compositions in LT than in ST fattening samples. However, the proportion between omega- 6 and omega -3 was significantly increased $(p=$ $0.0045)$ in ST. There were no significant differences in other fatty acid compositions between the 
Table 1. Carcass characteristics, blood, and fatty acid compositions of short-term and long-term fatten stage bovine steers

\begin{tabular}{|c|c|c|c|}
\hline Parameters & Short-term $(n=12)$ & Long-term $(n=10)$ & $p$-value \\
\hline \multicolumn{4}{|l|}{ Carcass traits } \\
\hline Carcass weight (kg) & $483.8 \pm 10.7$ & $460.1 \pm 14.2$ & ns \\
\hline Backfat thickness (mm) & $19.8 \pm 1.7$ & $10.1 \pm 0.8$ & $<0.001$ \\
\hline Marbling score & $6.2 \pm 0.1$ & $6.3 \pm 0.2$ & 0.195 \\
\hline Meat yield grade & $60 \pm 1.3$ & $67.7 \pm 0.7$ & $<0.001$ \\
\hline \multicolumn{4}{|l|}{ Blood analysis } \\
\hline Glucose (FBS) (mg/dL) & $120.30 \pm 7.83$ & $99.62 \pm 8.33$ & 0.0019 \\
\hline Insulin $(\mu \mathrm{g} / \mathrm{L})$ & $2.37 \pm 0.27$ & $8.59 \pm 1.64$ & 0.0015 \\
\hline Triglyceride (mg/dL) & $22.83 \pm 1.44$ & $21.00 \pm 2.13$ & 0.4899 \\
\hline HDL-cholesterol (mg/dL) & $120.00 \pm 0.00$ & $102.85 \pm 5.10$ & 0.0093 \\
\hline LDL-cholesterol (mg/dL) & $81.67 \pm 6.37$ & $31.38 \pm 2.34$ & $<0.001$ \\
\hline Cholesterol (mg/dL) & $174.83 \pm 10.04$ & $122.15 \pm 6.54$ & 0.0009 \\
\hline Total lipid (mg/dL) & $399.92 \pm 32.46$ & $295.85 \pm 13.69$ & 0.0058 \\
\hline Crude fat & $20.60 \pm 1.14$ & $18.97 \pm 1.39$ & ns \\
\hline \multicolumn{4}{|l|}{ Fatty acid (\%) } \\
\hline Myristic acid, C14:0 & $2.86 \pm 0.15$ & $2.87 \pm 0.16$ & ns \\
\hline Palmitic acid, C16:0 & $30.54 \pm 0.72$ & $28.55 \pm 0.7$ & ns \\
\hline Palmitoleic acid, C16:1n7 & $4.02 \pm 0.23$ & $4.51 \pm 0.28$ & ns \\
\hline Steric acid, C18:0 & $10.40 \pm 0.48$ & $11.15 \pm 0.49$ & ns \\
\hline Oleic acid, C18:1n9 & $48.47 \pm 1.06$ & $49.61 \pm 0.69$ & ns \\
\hline Vaccenic acid, C18:1n7 & $0.52 \pm 0.06$ & $0.45 \pm 0.04$ & ns \\
\hline Linoleic acid, C18:2n6 & $2.71 \pm 0.17$ & $2.28 \pm 0.16$ & ns \\
\hline y-Linolenic acid, C18:3n6 & $0.03 \pm 0.002$ & $0.04 \pm 0.03$ & 0.0367 \\
\hline a-Linolenic acid, C18:3n3 & $0.07 \pm 0.003$ & $0.08 \pm 0.008$ & ns \\
\hline Eicosenoic acid, C20:1n9 & $0.27 \pm 0.04$ & $0.32 \pm 0.04$ & ns \\
\hline Arachidonic acid, C20:4n6 & $0.08 \pm 0.006$ & $0.10 \pm 0.006$ & 0.0053 \\
\hline Adrenic acid, C22:4n6 & $0.03 \pm 0.002$ & $0.04 \pm 0.003$ & 0.0146 \\
\hline Saturated fatty acid (SFA) & $43.80 \pm 1.12$ & $42.57 \pm 0.91$ & ns \\
\hline Unsaturated fatty acid (USFA) & $56.20 \pm 1.12$ & $57.43 \pm 0.91$ & ns \\
\hline Monosaturated fatty acid (MUFA; Mono) & $53.28 \pm 1.07$ & $54.89 \pm 0.83$ & ns \\
\hline Polyunsaturated fatty acids (PUFA; Poly) & $2.91 \pm 0.17$ & $2.54 \pm 0.17$ & ns \\
\hline$\omega 3, n 3$ & $0.07 \pm 0.003$ & $0.08 \pm 0.008$ & ns \\
\hline$\omega 3, n 6$ & $2.84 \pm 0.17$ & $2.46 \pm 0.17$ & ns \\
\hline Ratio, n6/n3 & $40.70 \pm 1.86$ & $32.04 \pm 2$ & 0.0045 \\
\hline MUFA/SFA & $1.23 \pm 0.06$ & $1.30 \pm 0.05$ & ns \\
\hline PUFA/SFA & $0.07 \pm 0.005$ & $0.06 \pm 0.005$ & ns \\
\hline
\end{tabular}

All values are mean \pm SEM.

ns, not significant; FBS, fasting blood sugar; HDL, high-density lipoprotein; LDL, low-density lipoprotein; SEM, standard error of means.

two different groups.

The instability of blood levels such as insulin can lead to imbalance of protein and amino acid. Insulin is an anabolic hormone related to glucose and lipid metabolism. Feed intake and nutrient partitioning in ruminants are caused by insulin. Blood levels including glucose, cholesterol, HDL, LDL, and total lipid concentrations were affected by the different diets [13]. Nutritional manipu- 
lations and feeding period influence carcass growth and weight, and intramuscular fat content [7]. In particular, the modified energy diets and unsaturated fatty acids affect the fatty acid composition of the meat [14]. Therefore, differences in fat content between the two samples can be attributed to differences in blood levels and meat fatty acid composition. However, in this study, the ST and LT groups did not differ in body weight. The differences in backfat thickness and yield grade were observed for ST and LT. Fatty acid profiles have been widely studied to evaluate meat characteristic such as taste. Oleic acid is the most abundant fatty acid in beef, followed by palmitic and steric acids [15]. GLA synthesized by the essential amino acid linoleic acid (called the omega-6 fatty acid) is required for human health [16]. Essential fatty acids (EFAs) are required for function and development of brain, growth and development of muscle, and regulation of metabolism and reproductive processes [17]. ARA, a polyunsaturated fat belonging to omega-6 family, is an important fatty acid involved in the structure and function of human tissues, immune function, growth and function of brain and retina [18]. Furthermore, docosahexaenoic acid and ARA are typical fatty acid sources in the brain. AdA, a long-chain fatty acid, also belongs to the omega-6 family and is formed via the linoleic acid biosynthesis pathway. AdA is involved in alpha-linolenic acid (ALA) and linoleic acid (LA) metabolism as omega-3 fatty acid and omega-6, respectively [19]. The omega-6 and omega-3 polyunsaturated fatty acids (PUFAs) derived from food are one of the EFAs for humans because these cannot be synthesized in the human body [16]. PUFAs are an important fatty acid group involved in regulation of receptor activities and signal transduction on cell membranes. Recently, it has been shown that the $\mathrm{n} 6 / \mathrm{n} 3$ (omega-6/omega-3) ratio may provide a better understanding of human diseases such as heart disease and depression [20]. Omega-3 PUFAs also are required for growth and development of brain and retina, prevention of coronary artery, hypertension, and diabetes [21]. Thus, meat is a significant dietary source of PUFAs for human health and preventing diseases. Our observations showed that no significant changes were observed in the n6 and n3 contents. However, $\mathrm{n}-6 / \mathrm{n}-3$ ratio was significantly different $(p=0.0045)$. In this study, the obtained results were not enough for consideration. Further study may provide some evidence to improve human health via balanced $n-6 / n-3$ ratio in the managing period of beef and the diet provided to steers.

\section{Metabolite profiling using NMR spectroscopy analysis}

Meat metabolomics profiling was performed in ST- and LT fattening samples by NMR (Fig. 1). PLS-DA score plots revealed good clustering of the managing period based on qualitative and quantitative data (Fig. 1A). Metabolomics data were quantified with missing data of 4\%-5\%. The characteristics of the PLS model were sufficient, as follows: NMR $\left(R^{2} X=0.555, R^{2} Y=0.799\right.$, and $\left.\mathrm{Q}^{2}=0.503\right)$. PLS-DA loading plots showed differences in metabolite concentrations between the two groups (Fig. 1B). Twenty-nine metabolite compounds were obtained from ST and LT and data were validated by cross-validation analysis of variance and permutation test (data not shown). For the potential indicator selection between ST and LT, creatine, carnosine, lactate, succinate, carnitine, and glycerol were the most important metabolites for determining fattening period (Fig. 1C). Thus, these metabolites could be representative of the fattening stage in cattle. Among the six metabolites, lactate and creatine showed significantly increased levels in the ST group, while carnosine showed higher intensity in LT (VIP > 1, $p<0.05$; Fig. 1D).

Analysis of metabolomics data provides an understanding of the metabolic networks and biological systems involved in the process. In this study, NMR-based metabolomics analysis led us to identify unique molecular processes related to beef quality and taste-related metabolites in the different fattening stages. Because consumers, agriculture-related experts, scientists, and research institutes require reliable information to improve or maintain meat quality and taste, 
(A)

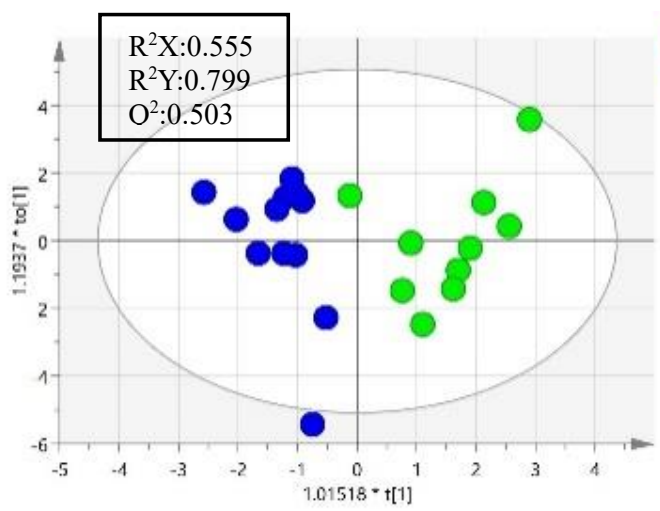

(B)

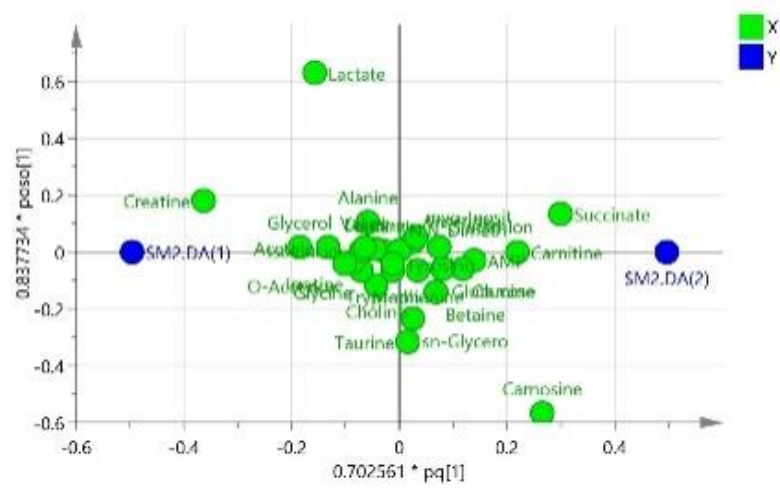

(C)



(D)



Fig. 1. Metabolite profiling for beef meat obtained from short-term and long-term fattening period samples. (A) PLS-DA score plot, (B) Loading plot of the PLS-DA model, (C) Variable importance in projection from OPLS-DA is indicated by red color (VIPs $>1)$, (D) Boxplots for the potential indicators and related mechanisms significantly differed by NMR (VIPs $>1, p<0.05)$. Short-term fattening group $(n=12)$, long-term fattening group $(n=10)$. Variations in the score plot were defined with a $95 \%$ confidence interval. PLS-DA, partial least squares-discriminant analysis; OPLS-DA, orthogonal partial least squares-discriminant analysis; NMR, nuclear magnetic resonance; VIP, variable importance in projection. 
we investigated short fattening period-related metabolites along with mineral, sensory, and fatty acid compositions. One of the identified metabolites, creatine, is a major compound in muscle energy metabolism. Increased creatine content-related sourness and bitterness in muscles may delay post-mortem lactate formation and the decrease in $\mathrm{pH}$ [22]. Similarly, the observed high concentrations of lactate as bitterness may be associated with increased citric acid cycle metabolism. High lactate concentrations may also indicate differences in energy metabolism such as in the glycolysis and gluconeogenesis pathways. In the arginine and proline metabolism and glycine, serine and threonine metabolism, creatine showed higher concentrations in the ST group. In the beta-alanine and histidine metabolism, carnosine had higher concentrations in the LT group. Carnosine is made up of the amino acids alanine and histidine, and is an essential dipeptide for muscle development, cognitive function, and preventing cell damage as an antioxidant [23,24]. Taken together, our study identified twenty-nine metabolites from ST and LT groups that could be considered as determinants of fattening stage. Thus, a combination of the managing period and different diets with metabolomics analysis enabled a more detailed evaluation of the fattening stage. The differences in amino acid profiles suggested that the biosynthesis and degradation of proteins might vary according to beef quality. The metabolomics analysis suggested that the specific metabolites could be potential indicators of taste. Carnosine, creatine, and lactate, taste-related metabolite compounds, were significantly expressed between the different fattening stages, while methionine, isoleucine, glycine, glutamine, alanine, leucine, taurine, and tyrosine showed no significant differences (data not shown). Thus, it is difficult to predict as of now, which candidate metabolites are important for the taste contributing to palatability.

\section{Sensory and mineral evaluation}

Mineral concentrations and taste scores were determined for the two different fattening stages (Figs. 2 and 3). Surprisingly, in this study, taste scores including SRS, STS, UMS, SWS, and BRS showed no differences between ST and LT samples. Mineral concentrations such as $\mathrm{Ca}$, $\mathrm{Cu}, \mathrm{Fe}, \mathrm{K}, \mathrm{Na}, \mathrm{Mg}$, and $\mathrm{Zn}$ significantly differed between $\mathrm{ST}$ and LT, while $\mathrm{Mn}$ and $\mathrm{P}$ showed no differences. The $\mathrm{Ca}, \mathrm{K}$, and $\mathrm{Na}$ concentrations were significantly increased in ST, whereas, $\mathrm{Cu}, \mathrm{Fe}, \mathrm{Mn}$, and $\mathrm{Zn}$ levels were higher in LT. These findings suggest that the combined differences in mineral concentrations did not lead to differences in the taste score between ST and LT groups.

The relevance of the electronic tongue for more rapid and sensitive screening of meat taste has become important. The palatability in beef also is generally attributed to tenderness, flavour, and/ or juiciness. The use of the electronic tongue for evaluating taste provides a better understanding of appropriate combinations of taste-related metabolites for developing beef quality, although many molecules still remain undetected due to the limitations of the methodology.

Calcium is the most abundant mineral in bones and is essential for bone development and prevention of osteoporosis [25]. Calcium not only reduces the fat uptake but also decreases the concentrations of LDL and total cholesterol [26]. Further, calcium is involved in dynamic signaling processes, including in taste cells. The instability of the atherosclerotic plaque is caused by the calcium accumulation [27]. The low concentrations of calcium can cause various symptoms, including hair and nail fragility and mood disorders such as irritability, depression, and sleepiness. The prolonged hypocalcaemia causes loss of consciousness. Copper mainly accumulates in heart, liver, muscles, and brain and is related to tremors, muscle stiffness, and trouble in speaking [28]. The excess copper levels in blood cause serious injury to the kidney, liver, and artery. Copper deficiency can leads to symptoms in circulatory, nervous, and digestive disorders by National Research Council (US) Committee [29]. Iron is an important mineral component of haemoglobin, myoglobin, and 


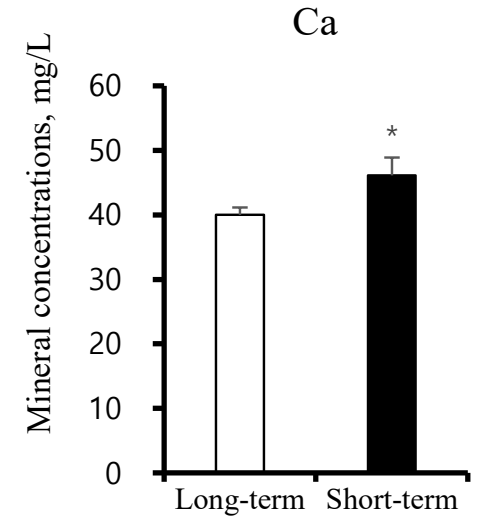

$\mathrm{Cu}$

$\mathrm{Fe}$
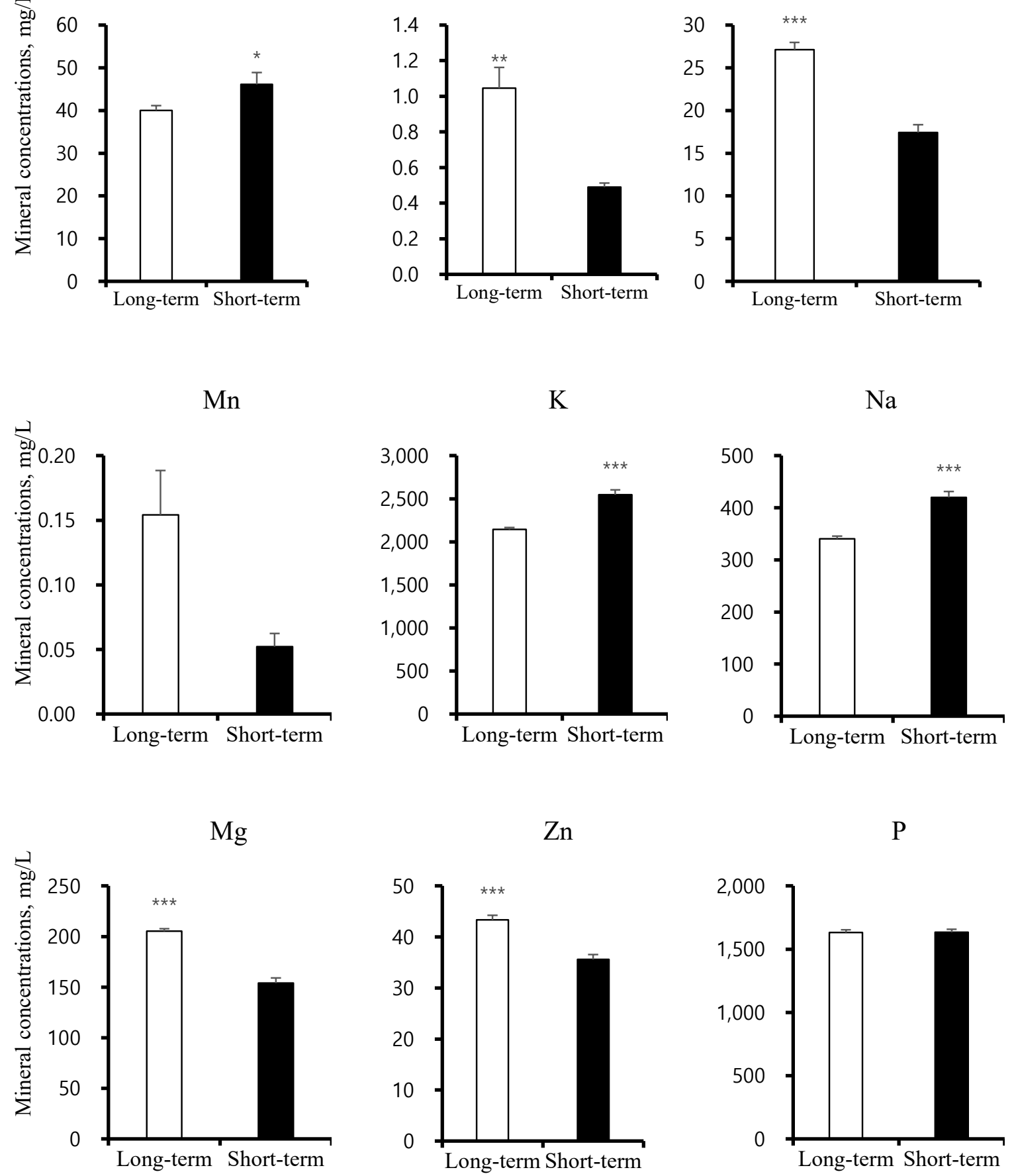

Fig. 2. Mineral concentration in beef meat from short-term and long-term fattening groups. ${ }^{*} p<0.05, "{ }^{* *} p<0.01,{ }^{* * *} p<0.001$.

various enzymes in human body. Iron deficiency can affects anemia and various symptoms involved in fatigue, weakness, and skin lesions due to poor diet, malabsorption, and blood loss [30]. Potassium can decrease the risk of high blood pressure and cerebral apoplexy [31]. Sodium mineral is the most common alkali metal and important for umami taste compound involved in mono-sodium glutamate (MSG) [32]. High concentrations of sodium are associated with high blood pressure and 
(A)

SRS

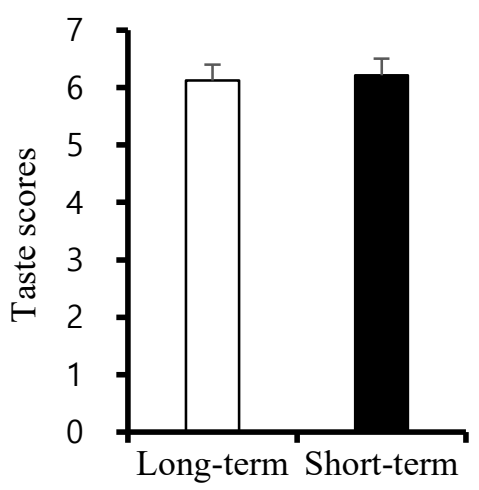

SWS



STS

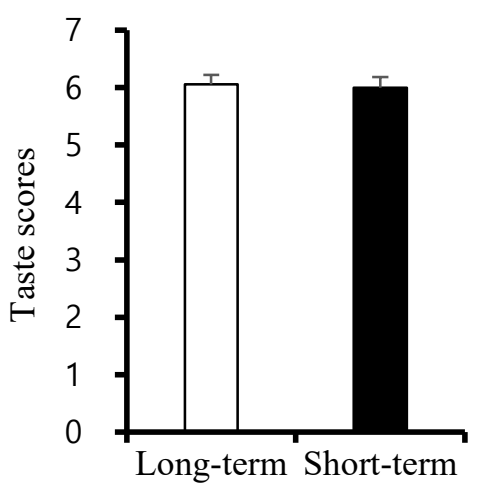

UMS

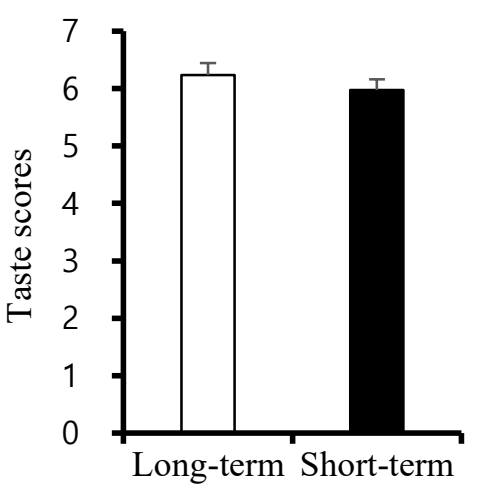

(B)

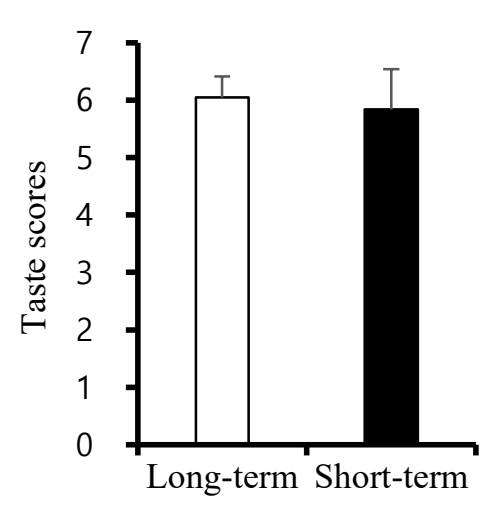

BRS
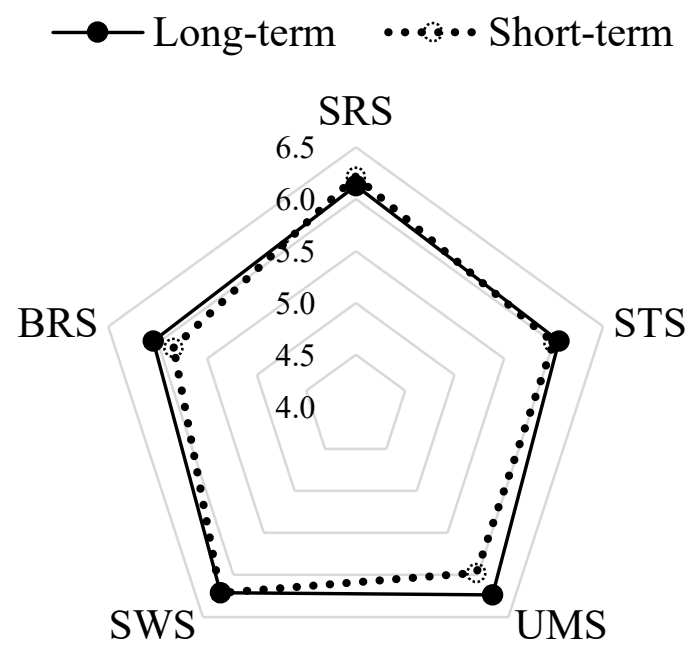

Fig. 3. Sensory evaluation by electronic tongue between beef meat from short-term and long-term fattening groups. (A) Taste score from each sensor of e-tongues, (B) The combination of sensory representation by radar graph. SRS, sourness; STS, saltiness; UMS, umami; SWS, sweetness; BRS, bitterness. 
arteriosclerosis [33]. Magnesium acts as a cofactor or an activator of several important enzymes and is essential for release of muscle tension and bone structure. Magnesium deficiency occurs mainly due to low food intake, and contributes to several symptoms in atherosclerosis, cardiovascular system, and metabolic disorders [34]. Zinc contributes to cellular metabolism and acts as an important modulator of the central nervous system. In particular, emotional stress and disorders is caused by hypozincemia. Zinc deficiency results from low food intake and affects growth, immune defense, cognitive function, and bone health. Therefore, zinc deficiency, similar to hypomagnesemia, can increase stress and reduce the quality of life by causing palpitations, dry skin, hair loss, depression, visual disturbance, hearing loss, and loss of taste and smell $[35,36]$. Our data suggests that beef containing appropriate mineral amounts could offer potential health benefits.

In conclusion, metabolomic profiling was performed to identify metabolic indicators of beef characteristics related to taste in ST and LT fattening period samples using NMR. Twenty-nine taste-related metabolites were detected. In particular, metabolites related to lactate for bitterness and creatine for sourness were significantly increased in ST fattening meat. Carnosine may act as a good signature of managing period. The taste score and related metabolites were similar for the different fattening periods, although blood, free fatty acid, and mineral concentrations were significantly different between the two samples. Shortening the breed period when maintaining the tastes and meat grades will reduce the total costs of beef production. In future studies, quantification of these potential indicators may have applications in the prediction of beef quality during the growing stage, as well as fattening stages. Metabolites may also act as good signature for phenotype parameters. Nevertheless, we still have limited knowledge of the role of metabolites and their regulation in meat quality and taste.

\section{REFERENCES}

1. McNeill SH. Inclusion of red meat in healthful dietary patterns. Meat Sci. 2014;98:452-60.

2. Goldansaz SA, Guo AC, Sajed T, Steele MA, Plastow GS, Wishart DS. Livestock metabolomics and the livestock metabolome: a systematic review. PLOS ONE. 2017;12:e0177675.

3. Meale SJ, Morgavi DP, Cassar-Malek I, Andueza D, Ortigues-Marty I, Robins RJ, et al. Exploration of biological markers of feed efficiency in young bulls. J Agric Food Chem. 2017;65:9817-27.

4. Gowda GAN, Raftery D. Biomarker discovery and translation in metabolomics. Curr Metabolomics. 2013;1:227-40.

5. Tian JS, Xia XT, Wu YF, Zhao L, Xiang H, Du GH, et al. Discovery, screening and evaluation of a plasma biomarker panel for subjects with psychological suboptimal health state using 1H-NMR-based metabolomics profiles. Sci Rep. 2016;6:33820.

6. Pighin D, Pazos A, Chamorro V, Paschetta F, Cunzolo S, Godoy F, et al. A contribution of beef to human health: a review of the role of the animal production systems. Sci World J. 2016;8681491.

7. De Brito GF, Ponnampalam EN, Hopkins DL. The effect of extensive feeding systems on growth rate, carcass traits, and meat quality of finishing lambs. Compr Rev Food Sci Food Saf. 2017;16:23-38.

8. Kegley EB, Ball JJ, Beck PA, BILL E. Kunkle interdisciplinary beef symposium: impact of mineral and vitamin status on beef cattle immune function and health. J Anim Sci. 2016;94:5401-13.

9. Orellana C, Peña F, García A, Perea J, Martos J, Domenech V, et al. Carcass characteristics, fatty acid composition, and meat quality of Criollo Argentino and Braford steers raised on forage 
in a semi-tropical region of Argentina. Meat Sci. 2009;81:57-64.

10. Hwang YH, Joo ST. Fatty acid profiles, meat quality, and sensory palatability of grain-fed and grass-fed beef from Hanwoo, American, and Australian Crossbred cattle. Korean J Food Sci Anim Resour. 2017;37:153-61.

11. Folch J, Lees M, Sloane Stanley GH. A simple method for the isolation and purification of total lipides from animal tissues. J Biol Chem. 1957;226:497-509.

12. Morrison WR, Smith LM. Preparation of fatty acid methyl esters and dimethylacetals from lipids with boron fluoride-methanol.J Lipid Res. 1964;5:600-8.

13. Griffin JD, Lichtenstein AH. Dietary cholesterol and plasma lipoprotein profiles: randomized-controlled trials. Curr Nutr Rep. 2013;2:274-82.

14. Wood JD, Enser M. Manipulating the fatty acid composition of meat to improve nutritional value and meat quality. In: Purslow PP, editor. New aspects of meat quality: from genes to ethics. Cambridge, UK: Woodhead Publishing; 2017. p. 501-35.

15. Araujo de Vizcarrondo C, Carrillo de Padilla F, Martín E. Fatty acid composition of beef, pork, and poultry fresh cuts, and some of their processed products. Arch Latinoam Nutr. 1998;48:354-8.

16. Simopoulos, AP. An increase in the omega-6/omega-3 fatty acid ratio increases the risk for obesity. Nutrients. 2016;8:128.

17. Kaur N, Chugh V, Gupta AK. Essential fatty acids as functional components of foods: a review. J Food Sci Technol. 2014;51:2289-303.

18. Markworth JF, Mitchell CJ, D’Souza RF, Aasen KMM, Durainayagam BR, Mitchell SM, et al. Arachidonic acid supplementation modulates blood and skeletal muscle lipid profile with no effect on basal inflammation in resistance exercise trained men. Prostaglandins Leukot Essent Fatty Acids. 2018;128:74-86.

19. Lee JM, Lee H, Kang S, Park WJ. Fatty acid desaturases, polyunsaturated fatty acid regulation, and biotechnological advances. Nutrients. 2016;8:E23.

20. Fernandes MF, Mutch DM, Leri F. The relationship between fatty acids and different depression-related brain regions, and their potential role as biomarkers of response to antidepressants. Nutrients. 2017;9:E298.

21. Coletta JM, Bell SJ, Roman AS. Omega-3 fatty acids and pregnancy. Rev Obstet Gynecol. 2010;3:163-71.

22. Farshidfar F, Pinder MA, Myrie SB. Creatine supplementation and skeletal muscle metabolism for building muscle mass- review of the potential mechanisms of action. Curr Protein Pept Sci. 2017;18:1273-87.

23. Blancquaert L, Everaert I, Missinne M, Baguet A, Stegen S, Volkaert A, et al. Effects of histidine and $\beta$-alanine supplementation on human muscle carnosine storage. Med Sci Sports Exerc. 2017;49:602-9.

24. Boldyrev AA, Stvolinsky SL, Fedorova TN, Suslina ZA. Carnosine as a natural antioxidant and geroprotector: from molecular mechanisms to clinical trials. Rejuvenation Res. 2010;13:156-8.

25. Price CT, Langford JR, Liporace FA. Essential nutrients for bone health and a review of their availability in the average North American diet. Open Orthop J. 2012;6:143-9.

26. Chai W, Cooney RV, Franke AA, Bostick RM. Effects of calcium and vitamin D supplementation on blood pressure and serum lipids and carotenoids: a randomized, double-blind, placebo-controlled, clinical trial. Ann Epidemiol. 2013;23:564-70.

27. Ott C, Jacobs K, Haucke E, Navarrete Santos A, Grune T, Simm A. Role of advanced glycation end products in cellular signaling. Redox Biol. 2014;2:411-29.

28. Chaudhry HS, Bhimji SS. Wilson disease. Treasure island, FL: StatPearls Publishing; 2017. 
29. National Research Council. Copper in drinking water. Washington, DC: National Academies Press; 2000.

30. Miller JL. Iron deficiency anemia: a common and curable disease. Cold Spring Harb Perspect Med. 2013;3:a011866.

31. Seth A, Mossavar-Rahmani Y, Kamensky V, Silver B, Lakshminarayan K, Prentice R, et al. Potassium intake and risk of stroke in women with hypertension and nonhypertension in the Women's Health Initiative. Stroke. 2014;45:2874-80.

32. Maluly HDB, Arisseto-Bragotto AP, Reyes FGR. Monosodium glutamate as a tool to reduce sodium in foodstuffs: technological and safety aspects. Food Sci Nutr. 2017;5:1039-48.

33. Lu H, Wu C, Howatt DA, Balakrishnan A, Charnigo RJ , Cassis LA, et al. Differential effects of dietary sodium intake on blood pressure and atherosclerosis in hypercholesterolemic mice. J Nutr Biochem. 2013;24:49-53.

34. Gröber U, Schmidt J, Kisters K. Magnesium in prevention and therapy. Nutrients. 2015;7:8199226.

35. Mitsuya H, Omata N, Kiyono Y, Mizuno T, Murata T, Mita K, et al. The co-occurrence of zinc deficiency and social isolation has the opposite effects on mood compared with either condition alone due to changes in the central norepinephrine system. Behav Brain Res. 2015;284:125-30.

36. Piao M, Cong X, Lu Y, Feng C, Ge P. The role of zinc in mood disorders. Neuropsychiatry. 2017;7:378-86. 